\title{
Universal Rapid Testing for SARS-CoV-2 in Obstetrical Patients at a Suburban New York Tertiary Medical Center: Prevalence and Patient Characteristics
}

\author{
Li BS ${ }^{1 *}$, Grimes CL $^{3}$, Clare CA ${ }^{1,2}$, Vicens- Villafana \\ $\mathrm{J}^{1}$, Kadesh $\mathrm{A}^{1}$ and Kasoff $\mathrm{M}^{\mathbf{2}}$ \\ ${ }^{1}$ Department of Obstetrics and Gynecology, New York \\ Medical College, USA \\ ${ }^{2}$ Department of Obstetrics and Gynecology, New York \\ City Health + Hospitals/ Metropolitan, USA \\ ${ }^{3}$ Departments of Obstetrics and Gynecology and Urology, \\ New York Medical College, USA \\ *Corresponding author: Bernice S. Li, Department of \\ Obstetrics and Gynecology, New York Medical College, \\ 100 Woods Road, Valhalla, NY 10595, USA, Tel: (917)214- \\ 1353, (212)423-6262/ (914) 594-2112; Fax: (914) 594- \\ 2111; E-mail: Bernice.li@wmchealth.org
}

Received: February 23, 2021; Accepted: March 11, 2021; Published: March 18, 2021

\begin{abstract}
Objective: To report the rate of COVID-19 positive cases among pregnant patients presenting to labor and delivery, and to further characterize the asymptomatic positive patients. To compare the patient characteristics between the positive and negative groups.
\end{abstract}

Methods: We performed a retrospective review on pregnant patients who presented to labor and delivery from April 10 to June 10, 2020 at our suburban New York tertiary medical center after the institution of a universal COVID-19 screening policy. Demographic and clinical data from electronic medical records were collected.

Results: A total of 130 pregnant patients underwent COVID-19 testing with Polymerase Chain Reaction (PCR) nasopharyngeal swabs. $14.6 \%$ of women were positive at the time of presentation, and among those, $25 \%$ presented with COVID-19 symptoms. Among the 130 patients, the asymptomatic COVID-19 carrier rate was $14.4 \%$. More patients in the COVID-19 positive group identified as Hispanic (65.2\% vs. $32.5 \%$ ) and were obese (39.1\% vs.18.7 \%). There was no difference in overall maternal profiles including maternal age, gestational age, antepartum steroid use, smoking status. Delivery mode sand adverse obstetrical outcomes (risks of chorioamnionitis/endometritis, need for blood transfusion, or hypertensive disorder) were not different between groups. There was no reported maternal death or maternal intubation/ventilation in our study.

Conclusion: During the first two months of universal COVID-19 testing, most COVID-19 positive patients were asymptomatic throughout the hospital stay. Around one in seven asymptomatic patients tested positive on presentation. Obesity and Hispanic ethnicity were associated withCOVID-19 infection among this pregnant population.

Keywords: COVID-19; SARS-CoV-2; Coronavirus; Pregnancy; Obesity; Race; Ethnicity

\section{Introduction}

A novel coronavirus SARS-CoV-2 (COVID-19), first detected in Wuhan, China in December 2019, has been detected in millions of people worldwide. The United States now has the highest numbers of test-confirmed cases. Being the largest and most densely populated area in the US, New York remains an epicenter for the pandemic, with by far the largest outbreak in the nation with over 574,072 confirmed cases and 26,225 death as of this writing [1]. Since the announcement in March 2020 of the first containment zone in New Rochelle, located in Westchester County, New York, Westchester County has remained a "hotspot" with 45,335 positive cases and a $4.6 \%$ positive rate among all tested as of November $15^{\text {th }}, 2020$ [1]. There were several small case-series with relatively short study periods [2-4] investigated the characteristics of COVID-19 positive pregnant patients at the earliest period of the pandemic, the disease course in pregnant patients, and the extent to which SARS-CoV-2 infection impacts pregnant women in a suburban region, remain an ongoing research area.

With the continuous growth of the disease rate globally and a sign of second wave of disease in New York State, we expected that more obstetrical patients were exposed to the novel virus with a large proportion of them being asymptomatic. Recent analysis has suggested that pregnant women with symptomatic infection were at increased risk for severe COVID-19 associated illness including intensive care unit admission, invasive ventilation, extracorporeal membrane oxygenation, and death [5].

In order to have a better understanding of the population we are serving and the associations between SARS-CoV-2 infection and adverse pregnancy outcomes, we aimed to report the rate of COVID-19 positive patients and rate of asymptomatic positive patients presenting to labor and delivery after the institution of a universal screening policy. We further aimed to characterize the asymptomatic positive patients and to compare the patient characteristics between the positive and negative test groups.

\section{Methods}

This is a retrospective chart review of patients triaged or admitted
Austin J Obstet Gynecol - Volume 8 Issue 3 - 2021

Submit your Manuscript | www.austinpublishinggroup.com

Li et al. (C) All rights are reserved
Citation: Li BS, Grimes CL, Clare CA, Vicens- Villafana J, Kadesh A and Kasoff M. Universal Rapid Testing for SARS-CoV-2 in Obstetrical Patients at a Suburban New York Tertiary Medical Center: Prevalence and Patient Characteristics. Austin J Obstet Gynecol. 2021; 8(3): 1172. 
to labor and delivery at Westchester Medical Center (WMC) between April $10^{\text {th }} 2020$ and June $10^{\text {th }} 2020$ who met criteria for COVID-19 testing based on a universal testing policy. WMC is a tertiary care referral center providing care in the Hudson Valley Region with about 1,200 deliveries and 300 maternal transfers annually. At WMC, universal Polymerase Chain Reactive (PCR) nasopharyngeal swab testing was implemented on April $10^{\text {th }}, 2020$. Universal testing policy required all pregnant persons to be screened for symptoms of COVID-19, and received the PCR nasopharyngeal test upon admission. This study was approved by Institutional review board from the New York Medical College (study ID 14274) and by the Clinical Research Institute at Westchester Medical Center.

Pregnant persons who presented to labor and delivery either with an obstetrical or medical complaint that warranted admission, triage patients who demonstrated COVID-19 symptomatology (fever/chill, muscle ache, upper respiratory symptoms, loss of smell/taste etc.) or were considered at risk per the institution's infectious disease protocol, and antepartum patients who required prolonged inpatient management were included. Non-pregnant patients, pregnant patients presented to triage unit who did not require admission and showed no COVID-19 symptomatology with negative screening questionnaires, and patients who declined rapid COVID-19 testing were excluded from the study.

Continuous data was described with means and standard deviations or medians and ranges, depending on the distribution of data (parametric vs. nonparametric). Categorical data was described with rates/proportions. Parametric continuous data was compared with student t-tests and non-parametric data with a Mann-Whitney test. Proportions were compared with a chi-square or Fischer exact test. Statistical significance was defined as $p<0.05$. A power analysis was not performed as this was a retrospective study with a fixed sample size.

\section{Results}

Over a two-month period, a total of 130 pregnant patients underwent COVID-19testing with PCR nasopharyngeal swabs. 19 patients were tested positive (14.6\%) at the time of presentation to WMC labor and delivery. There were four patients (3.1\%) who had a history of positive COVID-19 test (two tested positive at an outside hospital and two tested positive prior to April $10^{\text {th }}$ ), then subsequently tested negative on presentation to WMC. Among these four patients, one person was tested COVID-19 positive again two weeks later as an antepartum inpatient.

Of the 20 patients positive for COVID-19 while inpatients (including the one antepartum patient who was positive on re-testing), five patients (25\%) presented with COVID-19 symptoms. Of the 125 asymptomatic patients, 18 tested positive for COVID-19 (14.4\%), and all remained symptom-free throughout their hospitalizations.

Overall, our patient population had a median age of 31 years (range19-46), and presented at a median gestational of 34 weeks (range 12 to 41 weeks). $40 \%$ of our patients self-identified as Hispanic, 29.3\% as Black, 27.7\% as White, with mean Body Mass Indices (BMIs) of 32. Most patients (96.9\%) presented with an obstetric indication including labor, planned cesarean delivery, induction of labor and other pregnancy complications (Table 1).
There were more patients who self-identified as Hispanic in the COVID-19 positive group than in the negative group (Hispanic $65.2 \%$ vs. $32.5 \%$; White $4.4 \%$ vs. $28.9 \%, \mathrm{p}=0.029$ ). Compared with COVID-19 negative group, the COVID-19 positive group had a significantly higher percentage of obese patients (BMI>30, 39.1\% versus $18.7 \%, \mathrm{p}=0.033)$. A greater proportion of patients in the COVID-19 positive group (13.0\%) resided in the Bronx borough (which has one of the highest positive rate in New York, 5.1\%) than in the COVID negative group (4.7\%), although this was not statistically significant $(\mathrm{p}=0.518)$.

Apart from the findings mentioned above, the COVID-19 positive and negative groups shared similar patient profiles including age, gestational age on presentation, antepartum steroid use, smoking status, and influenza vaccination status. There was no difference in delivery modes between the two groups. Our data has not suggested that COVID-19 positive patients were more likely to present with obstetrical complaints such as preterm contractions or Preterm Premature Rupture of Membranes (PPROM) than the COVID-19 negative patients. However, they were more likely to present with initial complaints of suspected COVID-19 symptoms $(\mathrm{p}<0.001)$. There was no significant difference between the two groups with respect to adverse obstetrical outcomes (risks of chorioamnionitis/ endometritis, needs for blood transfusion, or hypertensive disorder). There were no reported maternal deaths, maternal intubation or ventilation in our study (Table 1).

Among the five symptomatic COVID -19 positive patients, two patients underwent emergent cesarean delivery due to worsened COVID symptoms (dyspnea, oxygen desaturation), and both resulted in the delivery of live born neonates and were discharged in stable condition postoperatively. Three patients were discharged home without delivery after their COVID-19 symptoms improved.

Among the asymptomatic COVID-19 positive patients, two patients were discharged without delivery after observation for obstetrical reasons (glycemic control and preterm contractions). Both patients subsequently had negative COVID-19 test results and underwent planned cesarean delivery at 39-week gestation, with uncomplicated postoperative course. All other patients delivered during the same hospital stay and among those, three patients underwent preterm delivery ( 32 to 34 -week gestation) due to nonreassuring fetal heart tracing or severe preeclampsia.

\section{Discussion}

\section{Principal findings}

Overall, the rate of COVID-19 positive patients presenting to our suburban tertiary medical center labor and delivery was $14.6 \%$ during the two-month period, and there was a high rate of asymptomatic positive COVID-19 patients.

Early published data indicates a low $(<3 \%)$ prevalence of positive COVID-19 test results among asymptomatic patients in pregnant populations outside of the highly endemic region of New York City [6,7]. As a suburban tertiary hospital outside of New York City, we found a much higher prevalence of positive COVID-19 test results among asymptomatic pregnant patients (about one in seven patients), more comparable to the prevalence $(13.5 \%)$ observed by Columbia University Irving Medical Center and Allen Hospital located in the 
Table 1: Baseline maternal demographic and clinical characteristics.

\begin{tabular}{|c|c|c|c|c|}
\hline Age (Median) & 31 & $32(5.2 \%)$ & $31(5.9 \%)$ & 0.5911 \\
\hline Race and Ethnicity & & & & 0.0292 \\
\hline White & $36(27.7 \%)$ & $1(4.4 \%)$ & $35(32.7 \%)$ & \\
\hline Hispanic & $52(40.0 \%)$ & $15(65.2 \%)$ & $37(34.6 \%)$ & \\
\hline Black & $38(29.3 \%)$ & $7(30.4 \%)$ & $31(28.9 \%)$ & \\
\hline Asian & $2(1.5 \%)$ & $0(0 \%)$ & $2(1.9 \%)$ & \\
\hline Other & $2(1.5 \%)$ & $0(0 \%)$ & $2(1.9 \%)$ & \\
\hline BMI & 32 & $35.0(9.2)$ & $32.5(7.4)$ & 0.3391 \\
\hline Gestational age (Median) & $34(12-41)$ & 37 & 36 & 0.5753 \\
\hline COVID-19 symptoms & $5(3.8 \%)$ & $5(21.7 \%)$ & 0/107 (0\%) & $<0.001^{2}$ \\
\hline Admission for obstetrical indications & $126(96.9 \%)$ & 19/23 (82.6\%) & 107/107(100\%) & $<0.001^{2}$ \\
\hline Labor/IOL & $44(33.8 \%)$ & $9(39.1 \%)$ & $35(32.7 \%)$ & 0.5552 \\
\hline Scheduled cesarean section & $17(13.1 \%)$ & $3(13.0 \%)$ & $14(13.3 \%)$ & 0.9962 \\
\hline PTL/PPROM & $32(24.6 \%)$ & $2(8.7 \%)$ & $30(28.0 \%)$ & 0.0512 \\
\hline Fetal Status & $4(3.1 \%)$ & $1(4.3 \%)$ & $3(2.8 \%)$ & 0.6972 \\
\hline Hypertensive disorder & $18(13.8 \%)$ & $2(8.7 \%)$ & $16(14.9 \%)$ & 0.4312 \\
\hline COVID-19 Symptoms & $5(3.8 \%)$ & $5(21.7 \%)$ & $0(0 \%)$ & $<.001^{2}$ \\
\hline Other * & $10(7.7 \%)$ & $1(4.3 \%)$ & $9(8.4 \%)$ & 0.5072 \\
\hline \multicolumn{5}{|l|}{ Maternal antepartum complications } \\
\hline HTN associated disorder (gHTN, cHTN, PEC) & $33(25.4 \%)$ & $5(21.7 \%)$ & $28(26.2 \%)$ & $0.658^{2}$ \\
\hline DM (pre- and gestational DM) & $18(13.8 \%)$ & $2(8.7 \%)$ & $16(14.9 \%)$ & 0.4312 \\
\hline Asthma & $20(15.4 \%)$ & $3(13.0 \%)$ & $17(15.9 \%)$ & 0.7322 \\
\hline Anemia & $15(11.5 \%)$ & $3(13.0 \%)$ & $12(11.2 \%)$ & 0.8032 \\
\hline Obesity & $29(22.3 \%)$ & $9(39.1 \%)$ & $20(18.7 \%)$ & 0.0332 \\
\hline Autoimmune conditions & $4(3.1 \%)$ & $1(4.3 \%)$ & $3(2.8 \%)$ & 0.6972 \\
\hline Antepartum steroid use & $60(46.2 \%)$ & $8(34.8 \%)$ & $52(48.5 \%)$ & 0.2572 \\
\hline Mode of delivery & & & & 0.8042 \\
\hline Vaginal delivery & $43(33.1 \%)$ & $9(39.1 \%)$ & $34(31.8 \%)$ & \\
\hline Cesarean section & $63(48.5 \%)$ & $9(39.1 \%)$ & $54(50.5 \%)$ & \\
\hline Discharge without delivery & $22(59.4 \%)$ & $5(21.7 \%)$ & $17(15.9 \%)$ & \\
\hline Cesarean following vaginal delivery (twin gestation) & $1(0.8 \%)$ & $0(0 \%)$ & $1(0.9 \%)$ & \\
\hline Undelivered & $1(0.8 \%)$ & $0(0 \%)$ & $1(0.9 \%)$ & \\
\hline Smoking & & & & 0.7042 \\
\hline Current & $11(8.5 \%)$ & $1(4.3 \%)$ & $10(9.3 \%)$ & \\
\hline Former & $7(5.4 \%)$ & $1(4.3 \%)$ & $6(5.6 \%)$ & \\
\hline Non-smoker & $112(86.1 \%)$ & $21(91.3 \%)$ & $91(85.0 \%)$ & \\
\hline Influenza vaccine status & & & & 0.9662 \\
\hline Yes & $59(45.4 \%)$ & $11(47.8 \%)$ & $48(44.9 \%)$ & \\
\hline No & $47(36.2 \%)$ & $8(34.8 \%)$ & $39(36.4 \%)$ & \\
\hline Unknown & $24(18.4 \%)$ & $4(17.4 \%)$ & $20(18.7 \%)$ & \\
\hline Housing status & & & & 0.6442 \\
\hline Private house & $56(43.1 \%)$ & $8(34.8 \%)$ & $48(44.9 \%)$ & \\
\hline Apartment & $71(54.6 \%)$ & $15(65.2 \%)$ & $56(52.3 \%)$ & \\
\hline Correction facility & $1(0.8 \%)$ & $0(0 \%)$ & $1(0.9 \%)$ & \\
\hline Unknown & $2(1.5 \%)$ & $0(0 \%)$ & $2(1.9 \%)$ & \\
\hline
\end{tabular}




\begin{tabular}{|c|c|c|c|c|}
\hline County of Residence & & & & 0.5182 \\
\hline Westchester & $87(66.9 \%)$ & $14(60.8 \%)$ & $73(68.22 \%)$ & \\
\hline Bronx & $8(6.2 \%)$ & $3(13.0 \%)$ & $5(4.67 \%)$ & \\
\hline New York & $1(0.8 \%)$ & $0(0 \%)$ & $1(0.9 \%)$ & \\
\hline Kings & $1(0.8 \%)$ & $0(0 \%)$ & $1(0.9 \%)$ & \\
\hline Orange & $13(10 \%)$ & $3(13.0 \%)$ & $10(9.3 \%)$ & \\
\hline Rockland & $10(7.7 \%)$ & $3(13.0 \%)$ & $7(6.5 \%)$ & \\
\hline Sullivan & $3(2.3 \%)$ & 0 & $3(2.8 \%)$ & \\
\hline Other (Putnam, Sussex (NJ), Ulster) & $7(5.4 \%)$ & 0 & $7(6.5 \%)$ & \\
\hline Adverse maternal outcomes & & & & 0.2252 \\
\hline Endometritis/Chorioamnionitis & $7(5.4 \%)$ & 0 & $7(6.5 \%)$ & \\
\hline Worsened COVID19 & $1(0.8 \%)$ & $1(4.3 \%)$ & $0(0 \%)$ & \\
\hline Blood transfusion & $7(5.4 \%)$ & $1(4.3 \%)$ & $6(5.6 \%)$ & \\
\hline Other ** & $7(5.4 \%)$ & 0 & $7(6.5 \%)$ & \\
\hline None & $100(76.8 \%)$ & $18(78.3 \%)$ & 82 (76.6\%) & \\
\hline
\end{tabular}

$1=$ mean (standard deviation), student t-test

$2=$ proportion (percentage), chi-square

$3=$ median (range), mann-whitney

Abbreviations: BMI: Body Mass Index; PTL: Preterm Labor; PPROM: Preterm Premature Rupture of Membrane; BP: Blood Pressure; gHTN: Gestational Hypertension; cHTN: Chronic Hypertension; PEC: Preeclampsia; IOL: Induction of Labor

*Admitted for glycemic control, antepartum surveillance of short cervix, or cerclage placement.

"theadache associated with post-spinal anesthesia, peripartum cardiomyopathy in the setting of preeclampsia, uterine rupture during Trial of Labor after Cesarean section (TOLAC).

New York City (NYC) [2], implying a high exposure risk that our patient population faced in the pandemic in NYC.

Most patients who were COVID-19 positive were asymptomatic at presentation. Universal COVID-19 Polymerase Chain Reaction (PCR) testing has been essential to identifying these asymptomatic carriers in order to ensure prompt isolation precautions on admission. None of symptomatic COVID patients in our study required ICU admission or ventilation support, although recent analysis has suggested that symptomatic pregnant women were at higher risk for severe COVID-19 associated illness compared to non-pregnant population [5]. Our study did not find an increased risk of adverse pregnancy outcome such as preterm labor, PPROM, hypertensive disorders, including preeclampsia and gestational hypertension among COVID-19 patients. However, studies with limited sample size have suggested increased risk of adverse pregnancy outcome in COVID-19 population [8].

\section{Clinical and research implications}

Higher BMI has been associated with a more critical course of COVID-19 infections and is an important attributing factor for mortality in the general population [9]. Some theories have proposed that adipose tissue and its association with Angiotensin-Converting Enzyme (ACE)-2 expressions might increase patient susceptibility to viral host cell entry and propagation [10]. From 1999 to 2010, the prevalence of obesity increased from $28.4 \%$ to $34.0 \%$ in women ages 20 to 39 years with a higher prevalence in non-Hispanic Black and Mexican American women [11]. This trend could potentially explain the higher percentage of Black and Hispanic patients observed in the COVID-19 positive group in our study. Analyses have also highlighted racial and ethnic disparities in risk for infection, disease severity and mortality rate among pregnant women, indicating a need to address potential risk factors in these populations $[5,12]$.

Compared to the patient population Breslin et al. [13] described in their study, which included most subjects who resided in the Bronx county and Upper Manhattan, our general population resided in Westchester county had a lower percentage of women self-identified as Black or Hispanic, a higher education level, a lower poverty rate and less public transportation use according to US census survey (2018).

\section{Strength and Limitations}

Although the majority of our patients resided in the Hudson Valley region (WMC is approximately 30 miles from New York City), there was likely an influx of COVID-19 carriers from New York City/Bronx County, since many of our patients commute to and from New York City on a daily basis. Given a large COVID-19 prevalence in New York, we were able to analyze a good proportion of diverse subjects. However, the COVID-19 positive cohort still remained small, making extrapolations on this group difficult; this was a limitation of the study. In addition, there was loss of followup of many asymptomatic COVID-19 positive patients post-delivery. These patients were not retested after being discharged from WMC and many patients may have preferred to follow up at a community clinic or hospital closer to home, which made following the disease course challenging in our retrospective study. As a single-center study performed in Westchester County, our findings may also not be generalizable to other hospital centers or areas.

\section{Conclusion}

As the situation with COVID-19 rapidly unfolds in the nation, 
some rural suburban areas have already seen the first surge of COVID-19 cases, and a possible second wave of cases of COVID-19 is also on the rise again in the United States. It is critical that obstetricians keep up to date on the transmission rates in their area and based on national guidance. The implementation of universal COVID-19 testing in our obstetric unit allowed the identification of high-risk groups in our patient populations, which help facilitate prompt infectious disease precautions, provide closer antepartum and intrapartum monitoring, and better guide neonatal management and hospital policy for mother-baby interaction.

More work should be done to identify the utility of providing resources to potentially at-risk communities as identified in our study. Strategies should be considered to address the disparate impact of COVID-19 on certain racial and ethnic groups, with emphasis on the management of asymptomatic patients who may be at risk of worsening community spread.

\section{References}

1. NYS Department of Health COVID-19 tracker.

2. Breslin N, Baptiste C, Gyamfi-Bannerman C, Miller R, Martinez R, Bernstein $\mathrm{K}$, et al. Coronavirus disease 2019 infection among asymptomatic and symptomatic pregnant women: two weeks of confirmed presentations to an affiliated pair of New York City hospitals. Am J Obstet Gynecol MFM 2020;2(2):100118.

3. Liu W, Wang Q, Zhang Q, Chen L, Chen J, Zhang B, et al. Coronavirus Disease 2019 (COVID-19) During Pregnancy: A Case Series. Preprints. 2020;2020020373

4. Chen H, Guo J, Wang C, Fan L, Yu X, Zhang W, et al. Clinical characteristics and intrauterine vertical transmission potential of COVID-19 infection in nine pregnant women: a retrospective review of medical records. Lancet. 2020;395(10226):809-15

5. Ellington S, Strid P, Tong VT, Woodworth K, Galang RR, Zambrano LD, et al. Characteristics of women of reproductive age with laboratory-confirmed SARS-CoV-2 infection by pregnancy status-United States, January 22-June 7, 2020. Morb Mortal Wkly Rep. 2020;69(25):769-75.

6. Campbell KH, Tornatore JM, Lawrence KE, Illuzzi JL, Sussman LS, Lipkind $\mathrm{HS}$, et al. Prevalence of SARS-CoV-2 Among Patients Admitted for Childbirth in Southern Connecticut. JAMA. 2020;323(24):2520-2.

7. Miller ES, Grobman WA, Sakowicz A, Rosati J, Peaceman AM. Clinical Implications of Universal Severe Acute Respiratory Syndrome Coronavirus 2 (SARS-CoV-2) Testing in Pregnancy. Obstet Gynecol. 2020;136(2):232-4.

8. Antoun L, Taweel N, Ahmed I, Patni S, Honest H. Maternal COVID-19 infection, clinical characteristics, pregnancy, and neonatal outcome: A prospective cohort study. Eur J Obstet Gynecol Reprod Biol. 2020;252:55962.

9. Peng YD, Meng K, Guan HQ, Leng L, Zhu RR, Wang BY, et al. Clinical characteristics and outcomes of 112 cardiovascular disease patients infected by 2019-nCoV. Zhonghua Xin Xue Guan Bing Za Zhi. 2020;48(6):450-5.

10. Caci G, Albini A, Malerba M, Noonan DM, Pochetti P, Polosa R. COVID-19 and Obesity: Dangerous Liaisons. J Clin Med. 2020;9(8):2511.

11. Flegal KM, Carroll MD, Ogden CL, Curtin LR. Prevalence and trends in obesity among US adults, 1999-2008. JAMA. 2010;303(3):235-41.

12. El Chaar M, King K, Galvez Lima A. Are black and Hispanic persons disproportionately affected by COVID-19 because of higher obesity rates? Surg Obes Relat Dis. 2020;16(8):1096-99.

13. U.S. Census Bureau. American Community Survey (ACS). 2018. 